\title{
Model-Based Improvements in the Treatment of Patients with Strabismus and Axial High Myopia
}

\author{
Robert Hoerantner, ${ }^{1,2}$ Thomas Kaltofen, ${ }^{3}$ Siegfried Priglinger, ${ }^{4}$ Christian-Michael Fock, ${ }^{1,5}$ \\ Michael Buchberger, ${ }^{3}$ and Thomas Haslwanter ${ }^{3,6}$
}

Purpose. Eye motility disorders with axial high myopia and an enlarged globe are often characterized by a hypotropia of the affected eye, usually referred to as heavy-eye syndrome. Based on an intuitive interpretation of magnetic resonance (MR) images, the cause of the hypotropia has typically been assigned to the rectus muscles. In this study, the hypothesis that the oblique muscles play an important role in the underlying biomechanical disorder of this type of strabismus was investigated.

Methods. The hypothesis was tested by (1) a retrospective analysis of surgical results in one patient with unilateral axial high myopia; and (2) MR images of orbital tissues in two further patients with unilateral axial high myopia.

REsults. MR images demonstrated a pattern of extraocular muscle path displacements similar to those described previously, but also a uniform decrease in the cross-sectional area of the inferior oblique muscles. Computer modeling required decreased inferior oblique contractility in addition to displaced extraocular muscle paths to recreate the observed motility pattern accurately.

Concuusions. Patients with axial high myopia regularly show a reduction in the diameter of the inferior oblique. The resultant reduction in muscle-strength is important for the correct explanation of this complex eye movement disorder. (Invest Ophthalmol Vis Sci. 2007;48:1133-1138) DOI:10.1167/ iovs.06-0769

$\mathrm{M}$ otility disorders caused by a myopic globe with high axial length were already known in the 19th century. In 1864 , Donders ${ }^{1}$ reported frequent divergence in highly myopic patients "whose elongated globes find difficulty in orientating

From the Departments of ${ }^{1}$ Ophthalmology and ${ }^{5}$ Radiology, Krankenhaus der Barmherzigen Schwestern (Good Sisters Hospital), Ried, Austria; ${ }^{2}$ Department of Ophthalmology, University Hospital Graz, Austria; the ${ }^{3}$ Department for Medical Informatics Upper Austrian Research GmbH, Hagenberg, Austria; ${ }^{4}$ Department of Ophthalmology, Krankenhaus der Barmherzigen Brüder (Good Brothers Hospital), Linz, Austria; and the ${ }^{6}$ Department of Medical Technology, Upper Austrian University of Applied Sciences, Linz, Austria.

The SEE ++ software system is sold commercially by the UAR Department for Medical-Informatics, which employs two of the authors of this paper. The UAR GmbH is a nonprofit organization funded by the local Upper Austrian government, and any earnings are reinvested into further development of the SEE ++ software system.

Submitted for publication July 7, 2006; revised August 25 and October 12, 2006; accepted January 8, 2007.

Disclosure: R. Hoerantner, None; T. Kaltofen, None; S. Priglinger, None; C.M. Fock, None; M. Buchberger, None; T. Haslwanter, None

The publication costs of this article were defrayed in part by page charge payment. This article must therefore be marked "advertisement" in accordance with 18 U.S.C. $\$ 1734$ solely to indicate this fact.

Corresponding author: Thomas Haslwanter, Upper Austrian University of Applied Sciences, Medical Technology, Garnisonstr. 21, A-4020 Linz, Austria; thomas.haslwanter@fh-linz.at. themselves other than in the orbital axes." One hundred years later, the same phenomenon was described by Duke-Elder ${ }^{2}$ in a patient with only one myopic, elongated eye. As such patients tend to present with concomitant vertical hypotropia, the syndrome was labeled heavy-eye syndrome. ${ }^{3}$ In these patients the eye is frequently limited in vertical excursions, and Hugonnier and Magnard ${ }^{4}$ suggested the designation "the nervous syndrome of high myopia." Because of the muscular etiology, this was later changed to myopic myositis. ${ }^{5}$

While the term heavy-eye syndrome is descriptive of the hypotropia, it does not describe the etiology. In fact, the term is misleading, because gravity does not play a role in rotation of the eye about its geometric axis

Based on anatomic investigations with CT (computed tomography) and MRI (magnetic resonance imaging), Kommerell ${ }^{6}$ speculated that heavy-eye syndrome was caused by dislocation of medial and lateral rectus muscle into a caudal position. He speculated that these translocations should pull the eye downward, resulting in the observed hypotropia. He also reported a nasal shift of the superior and inferior rectus, resulting in adduction of the eye. MRI studies of myopic patients by Krzizok and Schroeder ${ }^{7}$ seemed to support these speculations, indicating dislocations of the rectus muscles. These muscle dislocations were suggested to be responsible for the clinical symptoms of patients with heavy-eye syndrome.

Because of the complexity of the oculomotor system, the full effects of a muscle displacement can be hard to estimate. This difficulty is especially true for the oblique extraocular muscles, the action of which is strongly influenced by eye orientation. Our simulations of the oculomotor mechanics allowed us for the first time to simulate the effects of the suggested modifications of the eye movements. ${ }^{8-10}$ In this report, we describe these simulations, together with the results of our investigation in three patients with axial high myopia.

\section{Methods}

\section{Subjects}

The study included three patients with axial high myopia. According to the Declaration of Helsinki, all patients were informed before the operation about the surgical procedure, the imaging methods, and the research goals of the study, and gave their informed consent. Furthermore, the study was approved by the local ethics commission.

Patient (P)1, who presented with esotropia and hypotropia, was treated 5 years before the study with superior oblique tendon transposition to the superior pole of the globe, defined as $7 \mathrm{~mm}$ posterior to the medial insertion of the superior rectus muscle. This patient also underwent a 4-mm resection of the ipsilateral lateral rectus with superior transposition and $4-\mathrm{mm}$ recession of the ipsilateral medial rectus with inferior transposition (Fig 1).

We included two more patients with asymmetric high myopia in one eye (P2, P3), hypotropia, and convergent strabismus. These two patients were used for MRI investigations. As they have little visual acuity in the affected eyes, they have at their own request not undergone surgery so far. Details on the patients are presented in Table 1. 

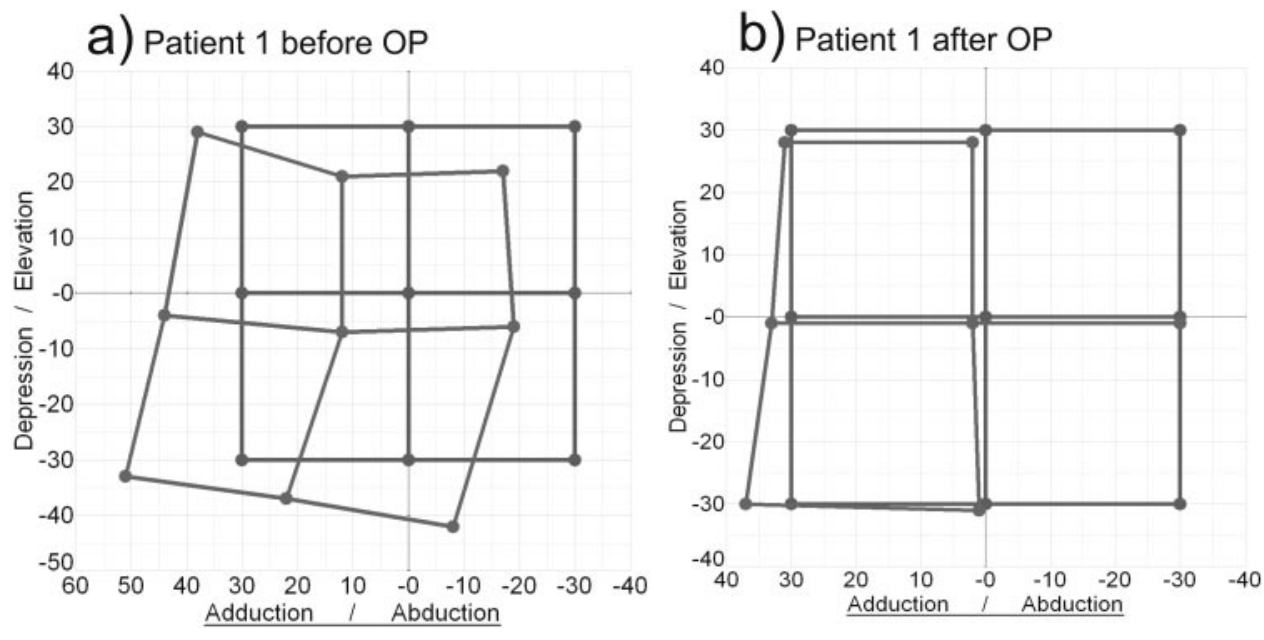

FigURE 1. Strabismus pattern for the right eye of P1, with left eye fixating. Adduction and abduction refer to the viewing direction of the following (right) eye. (a) Before the operation, showing the typical myopic eye-movement disorder. (b) After the operation, two fixation points (top right and bottom right) showed varying results in a prism cover test and Hess diagram and have been excluded from the diagram. All values are given in degrees.

\section{Magnetic Resonance Imaging}

The MR images of the oculomotor plant of P2 and P3 were obtained with a 1.5-Tesla gyro scanner (Intera; Philips, Eindhoven, The Netherlands). We used a circular head coil, type $\mathrm{C}$, and the images were $\mathrm{T}_{2}$-weighted/TSE (thick-slab sequence). To enhance the visibility of the image, gadolinium was administered. To ensure a constant eye position, a fixation point was presented straight ahead during the MR recording. Image resolution was $256 \times 256$ and $512 \times 512$, and the slice thickness varied from 1 to $4 \mathrm{~mm}$, depending on the sequence. Image planes were axial, coronal, and midsagittal. For navigation and exact muscle location, three dimensional (3-D) imaging was used (MRI software: Philips and OsiriX [open-source software]).

\section{Computer Model}

To simulate the effects of axial high myopia, we used two computer models: Orbit 1.8 (Eidactics, San Francisco, CA), and SEE ++ (Haslwanter et al. $\left.{ }^{10}\right)$. SEE ++ can simulate three different biomechanical models: One model tries to implement "active pulleys" such that pulley positions are modified as a function of gaze, as reported by Kono et al. ${ }^{11} \mathrm{~A}$ change in muscle forces affects gaze positions and influences pulley positions. However, no force calculations are considered for this implementation of the active-pulley hypothesis. The inferior oblique muscle is considered to have a pulley that moves anteroposteriorly with vertical gaze by half the distance of the inferior rectus muscle insertion. $^{11-13}$

The second biomechanical model in SEE ++ assumes passive pulleys: this approach is similar to the one in Orbit, but is implemented using different numerical and computational techniques. ${ }^{9,10}$ And the third model faithfully implements the biomechanical approach used by
Orbit 1.8. The simulations were run with the original Orbit system and with the three biomechanical models in SEE ++ . Orbit is able to simulate the myopic situation up to a $15-\mathrm{mm}$ globe radius and requires manual recalculation for pulley locations, muscle lengths, and so on, for larger globe radii. Therefore, we focused on the simulation of globes with a radius of $15 \mathrm{~mm}$, to be able to compare the results of the different models. SEE ++ can also simulate globes with a radius larger than $15 \mathrm{~mm}$.

\section{Results}

\section{Surgical Results}

When P1 presented at our clinic, she showed a typical myopic movement disorder (Fig 1a). Surgery involving the rectus and oblique muscles (described in the Methods section) eliminated the strabismus, as shown in (Fig 1b), without reducing the globe motility. ${ }^{14}$ The pattern for the left eye, with the right eye fixating, contained similar information and is not shown.

\section{Analysis of MRI Recordings}

In all highly myopic eyes (right eyes in P2 and P3), we found a consistent reduction of the thickness of the inferior oblique muscle. Figure 2 shows the results in P2: In the affected eye, the thickness of the inferior oblique was reduced to $0.9 \mathrm{~mm}$ (Fig. 2b), in contrast to that of the healthy eye $(2.3 \mathrm{~mm}$; Fig. 2a). The same pattern was observed in P3 (Figs. 3a, 3b). We also found the rectus muscle displacements that have previously been observed by other groups. ${ }^{7}$ The thickness of the

TABLE 1. Patient Characteristics

\begin{tabular}{|c|c|c|c|c|c|c|c|c|c|}
\hline Patient & Gender & $\begin{array}{l}\text { Age } \\
\text { (y) }\end{array}$ & $\begin{array}{l}\text { Horizontal } \\
\text { Deviation } \\
\text { (deg) }\end{array}$ & $\begin{array}{l}\text { Vertical } \\
\text { Deviation } \\
\text { (deg) }\end{array}$ & $\begin{array}{l}\text { Visual } \\
\text { Acuity }\end{array}$ & Globe Length & Surgery & MRI & Notes \\
\hline 1 & $\mathrm{~F}$ & 48 & +12 & 7.0 & $\begin{array}{l}0.4 \text { right } \\
0.5 \text { left }\end{array}$ & $\begin{array}{l}34.6 \mathrm{~mm} \text { right eye (US); } \\
30.0 \mathrm{~mm} \text { left eye (US) }\end{array}$ & Yes & No & $\begin{array}{l}\text { Myopia of the right eye } \\
\text { Rectus surgery and } \\
\text { surgery on the } \\
\text { oblique muscle }\end{array}$ \\
\hline 2 & $\mathrm{~F}$ & 36 & $\begin{array}{l}+25 \text { (high } \\
\text { variability) }\end{array}$ & 3.5 & $\begin{array}{l}0.32 \text { right } \\
0.4 \text { left }\end{array}$ & $\begin{array}{l}33.1 \mathrm{~mm} \text { right (MRI) } \\
28.9 \mathrm{~mm} \text { left (MRI) }\end{array}$ & No & Yes & Myopia of the right eye \\
\hline 3 & M & 29 & +6 & 3.5 & $\begin{array}{l}0.1 \text { right } \\
1.2 \text { left }\end{array}$ & $\begin{array}{l}34.0 \mathrm{~mm} \text { right (MRI \& US) } \\
22.2 \mathrm{~mm} \text { left (MRI \& US) }\end{array}$ & No & Yes & Myopia of the right eye \\
\hline
\end{tabular}

Age is at the time of the investigation. A positive $(+)$ horizontal deviation indicates esotropic deviation. Horizontal and vertical deviations are given for the primary position. Visual acuity and globe length (determined with US and MRI) are given for both eyes. 

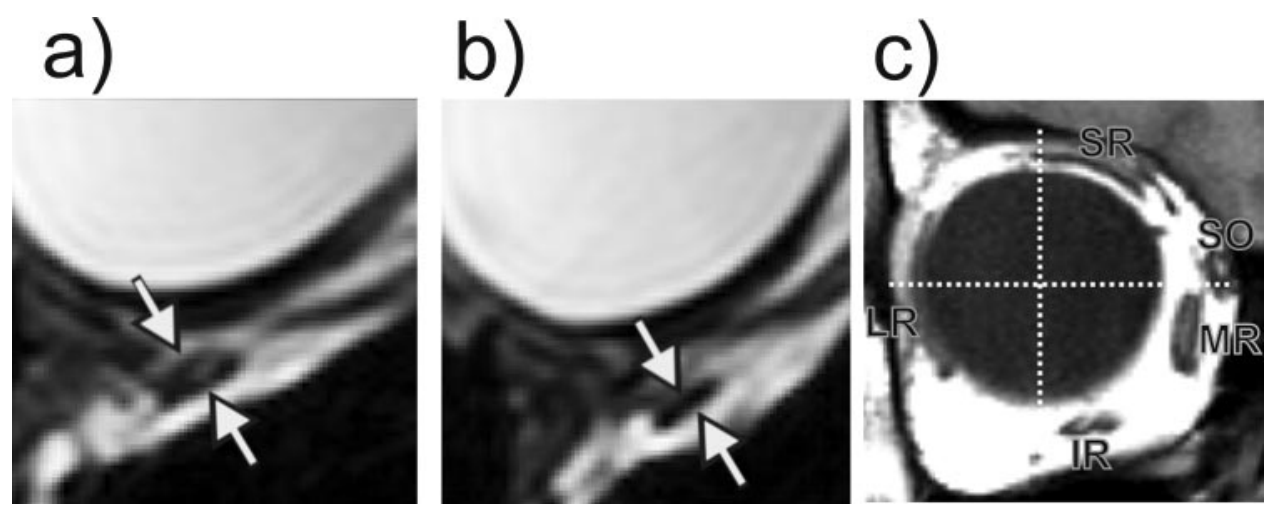

FIGURE 2. MRI results in P2: (a) The inferior oblique of the healthy left eye had a normal thickness (2.3 $\mathrm{mm}$ ) and a cross section of $17 \mathrm{~mm}^{2}$. (b) The inferior oblique of the myopic right eye had a reduced thickness $\left(0.9 \mathrm{~mm}\right.$ ) and a cross section of only $8 \mathrm{~mm}^{2}$. (c) Coronal view of the right eye (not perpendicular to orbital axis). All other muscles had a normal thickness, and typical dislocation, as reported by other groups. We found no indication of a significant approach of the lateral rectus to the inferior rectus muscle, or of parallel muscle paths. MR, medial rectus muscle; IR, inferior rectus muscle; LR, lateral rectus muscle; SR, superior rectus muscle; SO, superior oblique muscle.

rectus muscles, as well as of the superior oblique, was within the normal range ${ }^{15}$ (Fig. 2c). In both MRI studies, we found a length difference of 12 to $16 \mathrm{~mm}$ between the lengths of the medial and lateral rectus muscles, compared with the normal difference of $4 \mathrm{~mm}$. In P3, this is shown in Figure 3c. As the cross-sectional area of the inferior oblique can be path dependent, we measured the cross-section at different locations, and used the largest value. We ascertained that we had a correct orientation of the image plane by tracking the muscle center in adjacent image slices. Since the location of the muscle remains approximately unchanged, we are confident of using an appropriate image orientation.

\section{Simulations of Rectus Muscle Displacements}

The classic hypothesis is based on MR images showing that the lateral and medial rectus lie a bit under the distended globe, which may cause a depressive action (because they have slid under the globe equator), leading to hypodeviation (Fig. 4a). The observed medial displacement of the superior and inferior rectus is hypothesized to elicit esodeviation. ${ }^{6}$ To test this hypothesis, we started out with the simulation of a healthy subject, and increased the radius for the globe, from $11.2 \mathrm{~mm}$ to $15 \mathrm{~mm}$. Then, we dislocated the pulley position of the lateral and medial rectus pulleys by $3.4 \mathrm{~mm}$ into a more temporalcaudal position. (In their investigations, Krzizok et al., ${ }^{7}$ did not clearly distinguish between a shift of the insertion of the muscle on the globe and a shift of the muscle pulley.) The resultant eye positions indicate no hypotropia (shown in Fig. $4 \mathrm{~b}$; all diagrams correspond to the healthy eye fixating). Also the dislocation of all four rectus muscles pulleys, as suggested by Schroeder et al., ${ }^{16}$ produced no hypotropia (Fig. 4c).

\section{Simulation of the Effects of the Oblique Muscles}

To test the effect of the physiological changes that we found in the MRI recordings of $\mathrm{P} 2$ and $\mathrm{P} 3$, we started with the simulation of a healthy subject, and again increased the radius of the right globe to $15 \mathrm{~mm}$. In this simulation, we increased the length of the relaxed lateral rectus muscle by $8 \mathrm{~mm}$, as indicated in Figure 3c. The maximum thickness of the inferior oblique muscle, which is usually $2.4 \pm 0.4 \mathrm{~mm},{ }^{15}$ was between 0.9 and $1.3 \mathrm{~mm}$ in our patients (Figs. 2a, 2b, 3a, 3b). As investigations by other researchers have indicated that the muscle force depends linearly on the cross section, ${ }^{17,18}$ we decreased the active force of the inferior oblique muscle by a factor of 3, corresponding to an average reduction of the muscle cross-,section by approximately two thirds. (We refer to the muscle force generated by the innervation as active force, as opposed to passive force, which is caused by the elasticity of the muscle.) In addition, the observed displacement of the four rectus muscles was implemented, although with little effect on the final result of the simulation.

The effects of these changes are shown in Figure 5a. Note that for these simulations, as well as for those in Figure $5 \mathrm{c}$, we used only parameter changes that could be verified in the MRI. The results of the simulation are similar to the actually recorded eye positions in P1 before the operation (Fig. 1a): over the nine simulated fixation points, the horizontal-vertical deviation was $8^{\circ} \pm 4^{\circ}$ (mean $\pm \mathrm{SD}$ ), and $3^{\circ}$ for looking straight
FIGURE 3. MRI results of P3: (a) The inferior oblique of the healthy left eye had a normal thickness $(2.5 \mathrm{~mm})$ and a cross section of $22 \mathrm{~mm}^{2}$. (b) The inferior oblique of the myopic right eye had a reduced thickness $(1.3 \mathrm{~mm})$ and a cross-section of 5 $\mathrm{mm}^{2}$. (c) The myopic right globe in convergent position, after recalculation of the primary position, the length difference of the lateral and medial rectus muscle was $12 \mathrm{~mm}$ instead of $4 \mathrm{~mm}$, as found in healthy subjects.
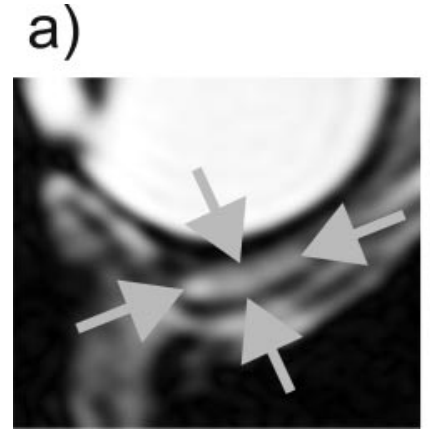

b)

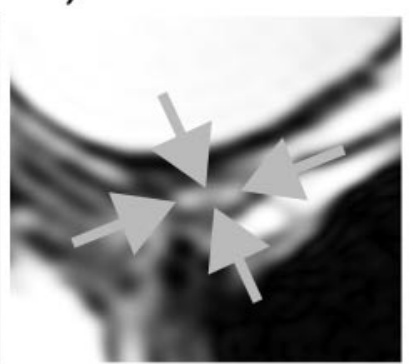

c)

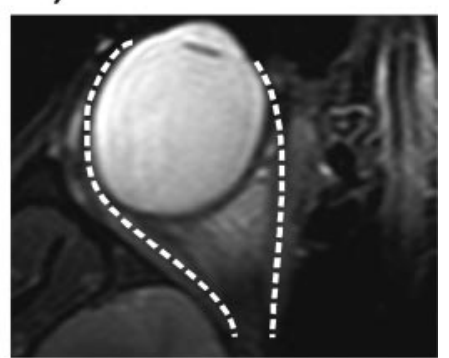


a) 3D - Model of SEE++

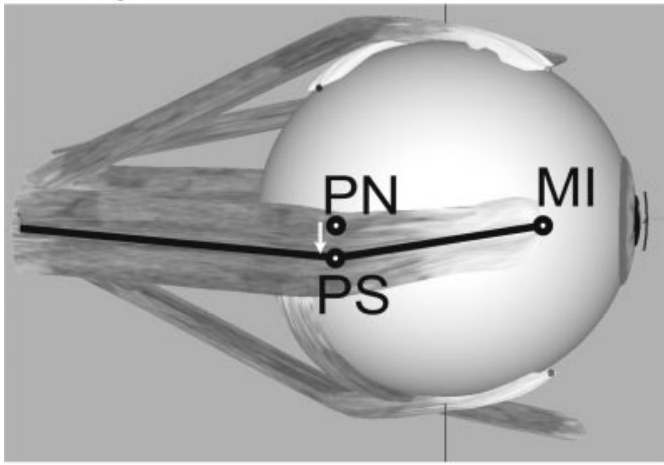

b) Lateral rectus muscle ${ }_{40}$ displacement

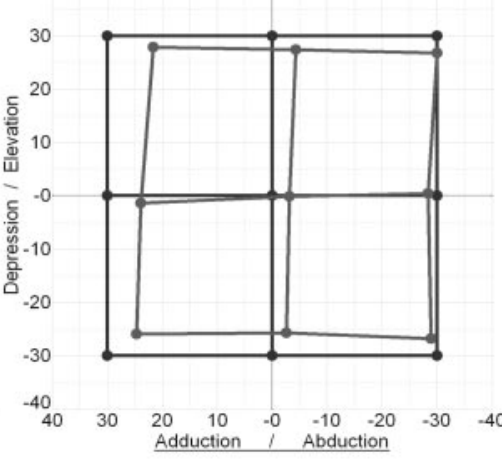

C) All rectus muscles displaced

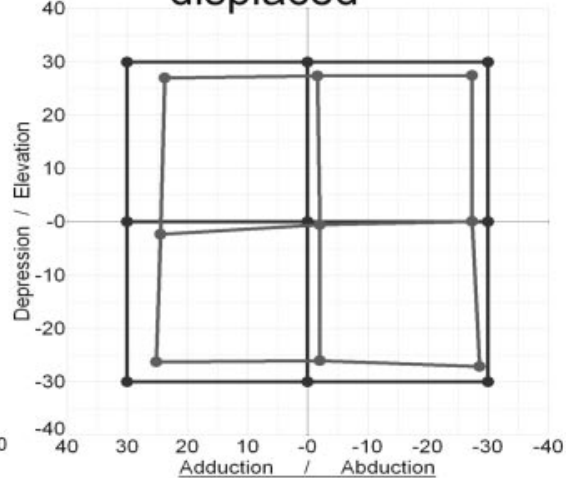

FiguRE 4. (a) Classic hypothesis for heavy-eye syndrome: displacement of the lateral rectus muscle into the temporal-caudal quadrant, as suggested by Kommerell. ${ }^{6}$ MI, muscle insertion; PN, normal pulley position. PS, Arrow: shifted pulley position. (b) SEE ++ simulation of displacement of lateral rectus pulley by $3.4 \mathrm{~mm}$ in temporal-caudal position, as described by Krzizok et al., ${ }^{7}$ with the healthy eye fixating at $\pm 30^{\circ}$ and a globe radius of $15 \mathrm{~mm}$. Adduction and abduction refer to the viewing direction of the following (right) eye, and all values are in degrees. (c) Simulation of displacement of all rectus muscle pulleys as described by Schroeder et al. ${ }^{16}$ and Krzizok et al. ${ }^{7}$ (LR, 2.9 mm down; SR, 1.5 mm medial; MR, $1.3 \mathrm{~mm}$ down; IR, $1.3 \mathrm{~mm}$ medial), globe radius $15 \mathrm{~mm}$. All simulation systems yielded the same results. (All screenshots from SEE ++ , using the active pulley model.)

ahead. By comparison, the eye positions that were obtained by changes in the rectus muscles alone (Fig. 4c) showed a deviation of $14^{\circ} \pm 3.5^{\circ}$, with a deviation of $14^{\circ}$ for looking straight ahead. Figure $5 \mathrm{c}$ shows the simulated strabismus pattern, after the after changes were made in the model: Recession of superior oblique and refixation to superior pole of globe, 4-mm recession and $4-\mathrm{mm}$ inferior shift of medial rectus, $4-\mathrm{mm}$ resection and superior shift of lateral rectus muscle (surgical parameters of P1).

Further improvements of the simulation results can be achieved by additional hypothetical changes in the models. The model parameters and results are shown in Figure 6a. Based on the eye movements observed in P1 before the operation (Fig. 1a), we investigated which surgical procedures would minimize the strabismus. The resulting simulations are presented in Figure 6 . The surgery with these parameters eliminated the strabismus almost completely, and the postop- erative results in the simulations (Fig. 6b) and in the patient (Fig. 1b) show a good similarity.

We obtained an interesting result when we simulated a bilateral increase in the globe's radius, combined with a reduction in the strength of both inferior obliques. In contrast to our simulations of a unilateral globe increase, simulations of a bilateral globe increase predicted no hypotropia. Hypotropia is defined by the relative position of one globe to the other. If both globes rotate downward and globe motility is not reduced, no hypotropia is measured in the Hess diagram.

\section{DisCuSSION}

The results of our MRI investigations of patients with axial high myopia, combined with our biomechanical simulations, indicate that the inferior oblique muscle plays an important role in

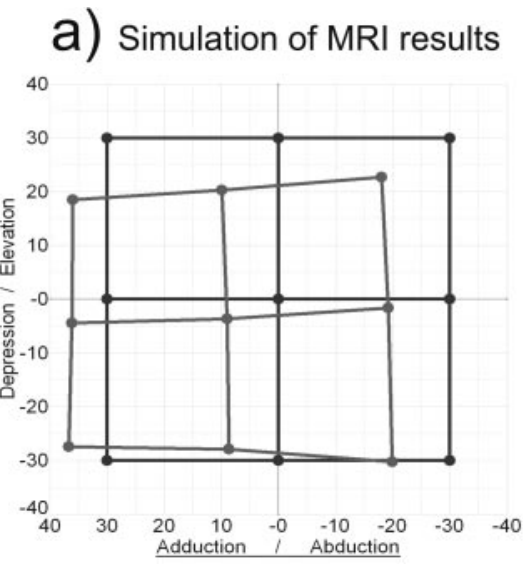

\section{b) 3D - Model of SEE++}

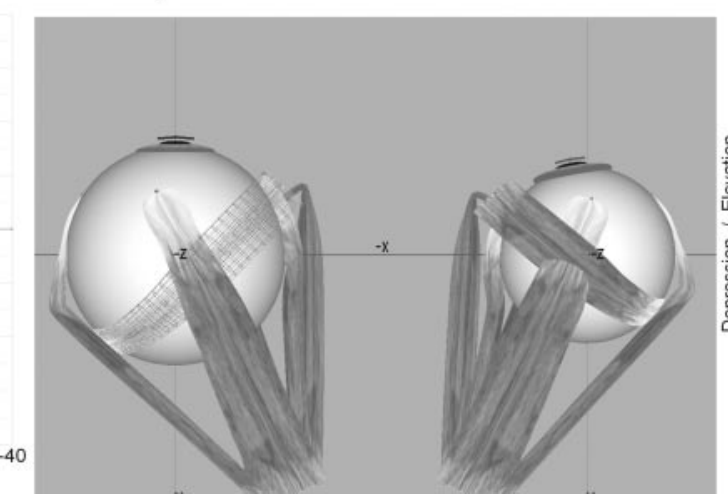

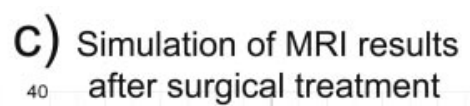

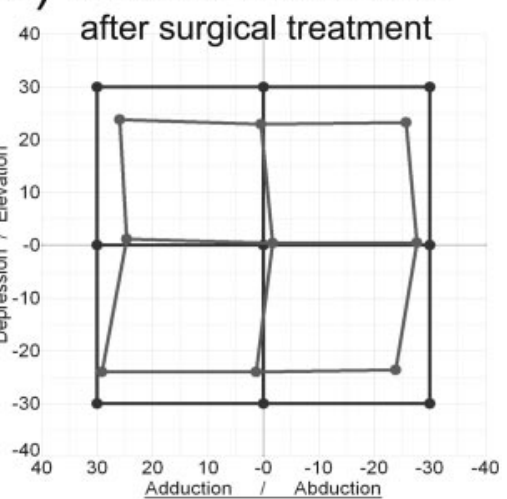

FiguRE 5. Simulation of MRI results. (a) Simulated heavy-eye syndrome, looking straight ahead and $\pm 30^{\circ}$ excentric. Simulation parameter of right eye: globe radius $15 \mathrm{~mm}$, inferior oblique force reduced to $33 \%$, rectus muscle pulley displacement as described by Schroeder et al., ${ }^{16}$ and lateral rectus muscle length increased by $8 \mathrm{~mm}$. Adduction and abduction refer to the viewing direction of the following (right) eye, and all values are in degrees. (b) Inferior view of the geometry of the oculomotor plant, inferior oblique muscle wireframed. (Screenshot from SEE ++ , active pulley model.) (c) Simulation of the surgical results of P1, based on the anatomy also used in (a). Parameters that were surgically changed are given in the text. All biomechanical models yielded very similar results. 
Figure 6. Simulation of the condition in P1. (a) Situation before surgery (active pulley model). Simulation parameters, right eye: globe radius, $17.15 \mathrm{~mm}$; contractile force of inferior oblique, $30 \%$; all rectus muscle pulleys displaced as described by Schroeder et al. ${ }^{16}$ : lateral rectus muscle length increased by 8 $\mathrm{mm}$, superior rectus muscle length increased by $3 \mathrm{~mm}$, insertion of superior oblique $x=13.4 \mathrm{~mm}, y=$ $-5.4 \mathrm{~mm}, z=9.9 \mathrm{~mm}$. Left eye globe radius $15 \mathrm{~mm}$. Adduction and abduction refer to the viewing direction of the following (right) eye, and all values are in degrees. (b). Simulation of the surgical results of P1, based on the anatomy also used for (a). Parameters that were surgically changed are given in the text. a) Simulation of patient 1 before OP SEE ++ active pulley

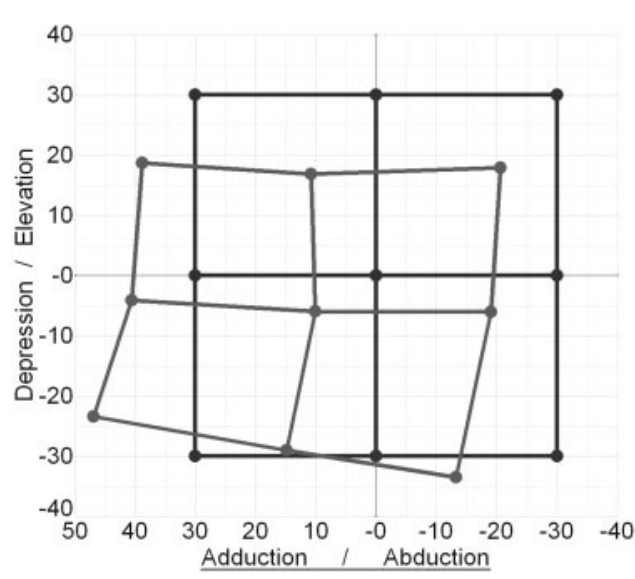

b)

Simulation of patient 1 after OP SEE++ active pulley the strabismus patterns observed in these patients. Previous investigations have interpreted a displacement of one or more muscles in a range of up to $3.4 \mathrm{~mm}$ as sufficient to explain the heavy-eye syndrome, ${ }^{19}$ and textbooks use a similar interpretation. ${ }^{6}$ In contrast, a high myopic globe radius, combined with a dislocation of the rectus muscles with up to $3.4 \mathrm{~mm}$, results in a change of the pulling direction of $12^{\circ}$, which hasaccording to our simulations-almost no effect. We found significant effects only for pulley dislocations of $7 \mathrm{~mm}$ or more, which we did not see in the MR images. Note that this effect is quite different from the active pulley movement, which occurs along the longitudinal axis of the eye muscle and which is also included in the simulations by SEE ++ . The $7-\mathrm{mm}$ shift considered herein is in the direction perpendicular to the muscle axis and is superposed on the active pulley movement.

According to our simulations, the main cause of the observed hypotropia is the reduced thickness of the inferior oblique muscle and the resultant reduction in muscle force. We speculate that the hypotropia of the inferior oblique reflects its special path, whereas the muscle belly of the four rectus muscles and of the superior oblique lies posterior to the globe and is thus less affected by an increase in the globe's diameter. The diameter of the inferior oblique may be reduced because of an increase in muscle length, with retention of normal muscle volume or because of some other unknown mechanism.

Although the movement of the human eye is controlled by six extraocular muscles, most explanations for oculomotor disorders focus on the four rectus muscles. However, the inclusion of the rectus muscles alone risks the omission of important contributions of the oblique muscles. As the history of the heavy-eye syndrome has shown, the complexity of the oculomotor plant makes it difficult to judge the precise effects of pathologic or surgical oculomotor changes by intuition, even if MRI images are available.

For the simulations presented herein, we used the computer models Orbit and SEE ++ . The simulation results show good correspondence with the experimentally recorded values. Implementing recent anatomic, MRI-based, and biomechanical findings, SEE ++ provides realistic simulations for all oculomotor disorders that we have investigated so $\mathrm{far}^{8}$ including the heavy-eye syndrome. In most cases, the different models give almost identical results.

In conclusion, patients with high myopia and the so-called heavy-eye syndrome may have inferior oblique atrophy in ad- dition to displaced extraocular muscle pulleys. Preoperative MRI can delineate both the amount of rectus EOM displacement and the presence of reduced inferior oblique cross-sectional area. Surgical planning to restore normal muscle paths can be combined with superior oblique weakening procedures and may improve surgical outcomes.

The data suggest that the reduction of the diameter of the inferior oblique muscle causes the main effect of the observed hypotropia in heavy-eye syndrome. Other muscle dislocations, as seen in the rectus muscles, have little or no effect on the recorded strabismus pattern.

\section{Acknowledgments}

The authors thank Joel Miller for providing the full source code of Orbit and for valuable feedback on the manuscript.

\section{References}

1. Donders FC. On the Anomalies of Accommodation and Refraction of the Eye, with a Preliminary Essay on Physiologic Dioptrics. London: The New Sydenham Society; 1864.

2. Duke-Elder WS. System of Ophthalmology: London: Henry Kimpton; 1969.

3. Ward DM. The heavy eye phenomenon. Trans Ophtbalmol Soc UK. 1967;87:717-726.

4. Hugonnier R, Magnard P. Statistics of 501 cases of oculomotor paralysis and diplopia. Bull Soc Opbtalmol Fr. 1960;73:80-99.

5. Hugonnier R, Magnard P. "Ocular paralysis" in Graves-Basedow disease. J Med Lyon. 1965;46:859-865.

6. Kommerell G. Störungen der Augen-, Lid- und Pupillenmotorik. In: Kaufmann H, ed. Strabismus. 2nd ed. Stuttgart, Germany: Ferdinand Enke Verlag; 1995:501-587.

7. Krzizok TH, Schroeder BU. Measurement of recti eye muscle paths by magnetic resonance imaging in highly myopic and normal subjects. Invest Opbthalmol Vis Sci. 1999;40:2554-2560.

8. Priglinger S, Buchberger M. Augenmotilitätsstörungen: Computerunterstützte Diagnose und Therapie Vienna: Springer; 2005.

9. Buchberger M. Biomechanical Modelling of the Human Eye. Thesis. Linz, Austria: Johannes Kepler University; 2004.

10. Haslwanter T, Buchberger M, Kaltofen T, Hoerantner R, Priglinger S. SEE + + : a biomechanical model of the oculomotor plant. Ann NY Acad Sci. 2005;1039:9-14.

11. Kono R, Clark RA, Demer JL. Active pulleys: magnetic resonance imaging of rectus muscle paths in tertiary gazes. Invest Ophthalmol Vis Sci. 2002;43:2179-2188. 
12. McClung JR, Allman BL, Dimitrova DM, Goldberg SJ. Extraocular connective tissues: a role in human eye movements? Invest Ophthalmol Vis Sci. 2006;47:202-205.

13. Demer JL, Oh SY, Poukens V. Evidence for active control of rectus extraocular muscle pulleys. Invest Ophthalmol Vis Sci. 2000;41: $1280-1290$

14. Priglinger S, Hametner H, Haslwanter T. Functional topography as a guideline for differential diagnosis of vertical eye movement disorders and oblique muscle surgery. Spektrum Augenheilkd. 2001; 15:16-26.

15. Moeller TB, Reif E. CT- und MRT-Normalbefunde. Stuttgart, Germany: Thieme Verlag; 1998.
16. Schroeder B, Krzizok T, Traupe H. Magnetic resonance imaging of the right extraocular muscle paths in healthy persons and in patients with high myopia (in German). Rofo Fortschr Geb Rontgenstr Neuen Bildgeb Verfahr. 1998;168:466- 473.

17. Veeger HEJ, van der Helm FC. Inertia and muscle contraction parameters for musculoskeletal modelling of the shoulder mechanism. J Biomech. 1991;24:615-629.

18. Wexler AS, Ding J, Stuart A. A mathematical model that predicts skeletal muscle force. IEEE Trans Biomed Eng. 1997;44:337348.

19. Krzizok T, Schroeder B. Quantification of recti eye muscle paths in high myopia. Strabismus. 2003;11:213-220. 